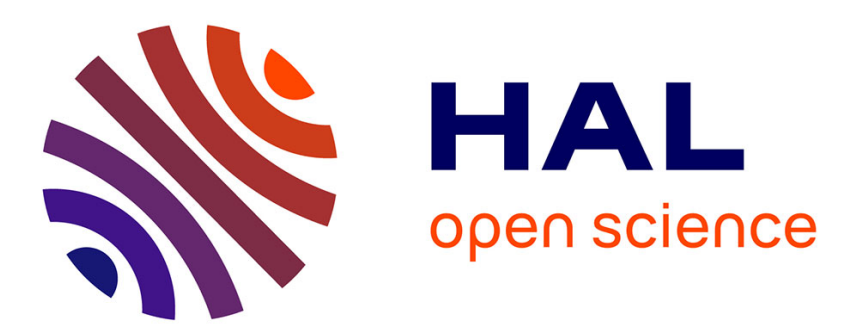

\title{
On the evolution of maximum turbulent kinetic energy production in a channel flow
}

\author{
F. Laadhari
}

\section{To cite this version:}

F. Laadhari. On the evolution of maximum turbulent kinetic energy production in a channel flow. Physics of Fluids, 2002, 14 (10), pp.L65-L68. 10.1063/1.1511731 . hal-02144585

\section{HAL Id: hal-02144585 \\ https://univ-lyon1.hal.science/hal-02144585}

Submitted on 9 Jun 2019

HAL is a multi-disciplinary open access archive for the deposit and dissemination of scientific research documents, whether they are published or not. The documents may come from teaching and research institutions in France or abroad, or from public or private research centers.
L'archive ouverte pluridisciplinaire HAL, est destinée au dépôt et à la diffusion de documents scientifiques de niveau recherche, publiés ou non, émanant des établissements d'enseignement et de recherche français ou étrangers, des laboratoires publics ou privés. 


\title{
LETTERS
}

The purpose of this Letters section is to provide rapid dissemination of important new results in the fields regularly covered by Physics of Fluids. Results of extended research should not be presented as a series of letters in place of comprehensive articles. Letters cannot exceed four printed pages in length, including space allowed for title, figures, tables, references and an abstract limited to about 100 words. There is a three-month time limit, from date of receipt to acceptance, for processing Letter manuscripts. Authors must also submit a brief statement justifying rapid publication in the Letters section.

\section{On the evolution of maximum turbulent kinetic energy production in a channel flow}

\author{
F. Laadhari \\ Laboratoire de Mécanique des Fluides et d'Acoustique, UMR CNRS 5509, EC de Lyon-UCB Lyon 1-INSA \\ de Lyon, 36 Avenue de Collongue, 69134 Ecully Cedex, France
}

(Received 3 June 2002; accepted 31 July 2002; published 6 September 2002)

\begin{abstract}
The Reynolds number effects on turbulent kinetic energy production and mean transport terms in near-wall turbulent channel flow are investigated analytically and with the help of direct numerical simulations (DNS). Using the momentum equation for turbulent channel flow, an analytical expression for the envelope of turbulent kinetic energy production curves is derived. It is shown that this envelope coincides with the wall-normal position at which the turbulent and viscous shear stress are equal. The DNS results carried out corroborate this finding and assess other quantitative details, namely the evolution of the peak of kinetic energy production and of its wall-normal position in terms of the Reynolds number. Empirical relations for the envelopes of the mean momentum transport terms and for their extrema position are also derived. (C) 2002 American Institute of Physics. [DOI: 10.1063/1.1511731]
\end{abstract}

During the two last decades, direct numerical simulations have provided databases that enable detailed studies of statistical properties in turbulent wall-bounded flows. In particular, the influence of the Reynolds number on statistical turbulence quantities has been examined for channel and boundary layer flows using these databases. ${ }^{1-3}$ The main conclusion is that turbulence statistics do not scale with the wall variables $\nu$ and $u_{\tau}$, where $\nu$ is the kinematic viscosity and $u_{\tau}$ the friction velocity which is defined as $u_{\tau}$ $=\left(\tau_{w} / \rho\right)^{1 / 2}$, with $\rho$ the density of the fluid and $\tau_{w}$ the mean shear stress at the wall.

The aim of the present study is to investigate the Reynolds number dependence of the production of mean turbulent kinetic energy $k=\frac{1}{2}\left(\overline{u^{2}}+\overline{v^{2}}+\overline{w^{2}}\right)$ in a channel flow [where $u, v, w$ denote the velocity fluctuations in the streamwise $(x)$, wall-normal $(y)$ and spanwise $(z)$ directions, respectively]. The understanding of such dependencies is important because it can give more insight on the mechanisms which govern the turbulent production.

Despite the impressive available direct numerical simulation (DNS) databases, new channel flow simulations have been conducted in order to obtain more Reynolds number cases covering a wider range. The numerical simulations are based on a pseudo-spectral code using Chebychev-tau formulation in the wall-normal direction and Fourier expansion in the streamwise and spanwise directions where periodic boundary conditions are applied. ${ }^{4}$ The parameters of the simulations are given in Table I. The computational domain has a size of $L_{x}^{+} \times 2 h \times L_{z}^{+}$(the superscript + denotes normalization by $u_{\tau}$ and $\nu$ ). The first mesh point away from the wall is at $\Delta y_{\min }^{+}<0.05$, while the grid-size in the streamwise direction is $\Delta x^{+}<10$ and in the spanwise direction $\Delta z^{+}$ $<4$.

For turbulent channel flow the mean momentum equation is exactly

$$
-\overline{u v}^{+}+S^{+}=1-\left(y^{+} / R^{+}\right),
$$

where $S^{+}=d \bar{U}^{+} / d y^{+}$and $R^{+}=u_{\tau} h / \nu, h$ being the channel half-width and $\bar{U}^{+}$the mean streamwise velocity. The second term of the right-hand side of (1) originates from the streamwise driving pressure gradient

$$
\partial \Pi^{+} / \partial x^{+}=-1 / R^{+} .
$$

The production rate of turbulent kinetic energy $P^{+}$ $=-\overline{u v}^{+} S^{+}$can be written as

$$
P^{+}=S^{+2}+S^{+}\left(-2 S^{+}+1-\left(y^{+} / R^{+}\right)\right) .
$$

It follows from Eqs. (1) and (2) that, at the position where the turbulent shear stress $-\overline{u v}^{+}$is equal to the viscous stress $S^{+}$, the production rate is given by

$$
P_{E}^{+}=S_{E}^{+2}=\frac{1}{4}\left(1-\left(y_{E}^{+} / R^{+}\right)\right)^{2},
$$

and depends, for a given Reynolds number, only on its normal position $y_{E}^{+}$and reaches an upper limit $P^{+}=\frac{1}{4}$ at large Reynolds numbers.

It should be noted that Eq. (3) represents the equation of the envelope of the curves of $P^{+}$for different values of $R^{+}$. Using Eq. (2) and expressing $S^{+}$and $P^{+}$as function of the independent parameters $R^{+}$and $\tilde{y}=y^{+} / R^{+}$, the envelope equation, given by 
TABLE I. Simulation parameters of the present DNS.

\begin{tabular}{rrrrrrrr}
\hline \hline$R_{e}{ }^{\mathrm{a}}$ & \multicolumn{1}{c}{$R^{+}$} & \multicolumn{1}{c}{$L_{x}^{+}$} & \multicolumn{1}{c}{$L_{z}^{+}$} & $\Delta x^{+}$ & $\Delta y_{\min }^{+}$ & $\Delta y_{\max }^{+}$ & $\Delta z^{+}$ \\
\hline 1263 & 72 & 909 & 454 & 7.1 & 0.022 & 1.8 & 3.6 \\
1566 & 90 & 846 & 376 & 6.6 & 0.027 & 2.2 & 2.9 \\
2125 & 120 & 1130 & 502 & 7.8 & 0.029 & 2.6 & 3.5 \\
2890 & 160 & 1505 & 669 & 7.8 & 0.021 & 2.6 & 3.5 \\
3295 & 180 & 1697 & 754 & 8.8 & 0.024 & 2.9 & 3.9 \\
4432 & 235 & 2216 & 985 & 8.7 & 0.018 & 2.9 & 3.8 \\
12606 & 589 & 2774 & 1387 & 7.2 & 0.044 & 7.2 & 3.6 \\
\hline \hline
\end{tabular}

${ }^{\mathrm{a}}$ Reynolds number based on centerline velocity and $h$.

$$
\mathrm{d} P^{+} / \mathrm{d} R^{+}=\left(\mathrm{d} S^{+} / \mathrm{d} R^{+}\right)\left(-2 S^{+}+1-\tilde{y}\right)=0,
$$

leads to $S^{+}=\frac{1}{2}(1-\tilde{y})$ and finally to Eq. (3). This result is of great importance as it shows that the Reynolds number effect on the rate of turbulence production, nondimensionalized by wall variables, can be inferred from the evolution of $y_{E}^{+}$.

Sahay and Sreenivasan ${ }^{5}$ looked at the evolution of $y_{E}^{+}$in the form $y_{E}^{+}=y_{E_{\infty}}^{+}+f_{E}\left(R^{+}\right)$where $y_{E_{\infty}}^{+}=\lim _{R^{+} \rightarrow \infty} y_{E}^{+}$and $f_{E}\left(R^{+}\right)$are both unknown. Using experimental data for various channel and pipe flows, they deduced an average value of $y_{E_{\infty}}^{+}=11$ and found a nonmonotic evolution for $f_{E}\left(R^{+}\right)$. This behavior is inconsistent with the monotonic increase of production with increasing Reynolds number. ${ }^{2,3}$ To clarify this behavior, we calculate $f_{E}$ for $y_{E_{\infty}}^{+}=11$ from the present DNS results and the datasets listed in Table II. The results are plotted in Fig. 1 where are also reported the data used by Sahay and Sreenivasan. ${ }^{5}$ The DNS data for channel flow show a monotonic decrease for $f_{E}$ with increasing Reynolds number. A power law fitting of these data gives

$$
y_{E}^{+} \approx 11+3454 R^{+-3 / 2} \text {. }
$$

Let us now consider the position of maximum production in channel flow. Upon differentiating $P^{+}$with respect to $y^{+}$, the position $y_{P}^{+}$of the peak production occurs when

$$
\frac{-\overline{u v}}{S^{+}}=\frac{\frac{\mathrm{d} \overline{u v}}{\mathrm{~d} y^{+}}}{\frac{\mathrm{d} S^{+}}{\mathrm{d} y^{+}}}=1+\frac{1}{R^{+} \frac{\mathrm{d} S^{+}}{\mathrm{d} y^{+}}}
$$

and for the maximum value

TABLE II. Parameters of the turbulent channel DNS datasets used for comparison.

\begin{tabular}{lcl}
\hline \hline & $R_{e}$ & $R^{+}$ \\
\hline Moser et al. (Ref. 6) & 3260 & 178 \\
& 7900 & 392 \\
Kawamura et al. (Refs. 7, 8) & 12485 & 587 \\
& 3330 & 180 \\
Iwamoto et al. (Ref. 9) & 8090 & 395 \\
& 13980 & 640 \\
& 1917 & 109 \\
& 2689 & 150 \\
& 5788 & 298 \\
& 7988 & 396 \\
\hline \hline
\end{tabular}

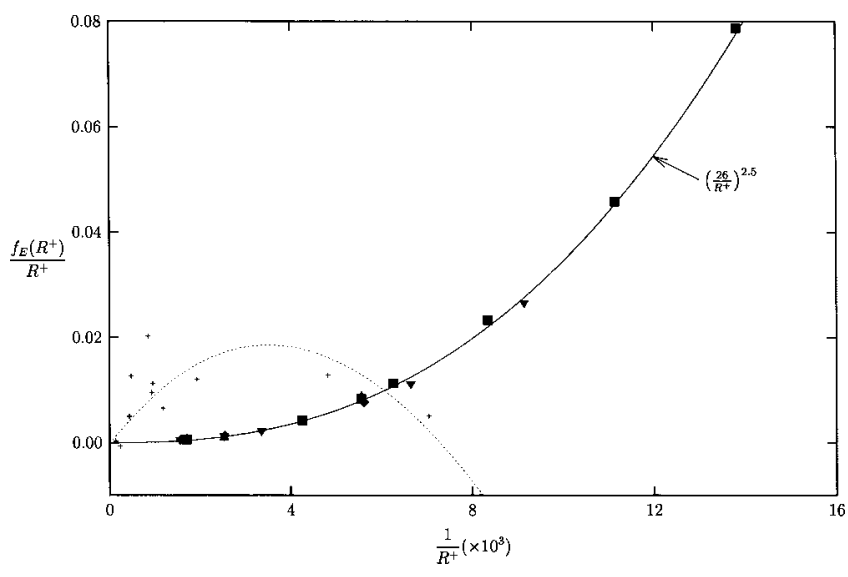

FIG. 1. Variation of $f_{E}\left(R^{+}\right) / R^{+}$with $1 / R^{+}$for $y_{E_{\infty}}^{+}=11$. Channel flow: present study; $\bullet$, Moser et al.; $\boldsymbol{\Lambda}$, Kawamura et al.; $\boldsymbol{\nabla}$, Iwamoto et al. $(+)$ Data used by Sahay and Sreenivasan; $\cdots$, their fitting curve.

$$
P_{P}^{+}=S_{P}^{+2}\left(1+\frac{1}{\left.\frac{\mathrm{d} S^{+}}{\mathrm{d} \tilde{y}}\right|_{\tilde{y}_{P}}}\right) \text {. }
$$

In the limit $R^{+} \rightarrow \infty$ the two positions, $y_{E}^{+}$and $y_{P}^{+}$, coincide at the location where $P^{+}=\frac{1}{4}$ and $-\overline{u v}^{+}=S^{+}=\frac{1}{2}$.

In the case of finite Reynolds number, because the viscous stress decreases monotonically with $y^{+}\left(\mathrm{d} S^{+} / \mathrm{d} \tilde{y}<0\right)$ and the peak position occurs for $-\overline{u v}^{+}<S^{+}<\frac{1}{2}$, the variation with Reynolds number of the normalized production peak is mainly related to the variation of the turbulent stress. This also implies that the peak position $y_{P}^{+}$is such that $y_{P}^{+}$ $<y_{E}^{+}$as shown in Fig. 2, where are plotted the evolutions of $P^{+}, \overline{u v}+2$ and $S^{+2}$ with $y^{+}$for $R^{+}=72$.

Equation (6) can be rewritten as

$$
P_{P}^{+}=S_{P}^{+2}\left(1+g\left(\tilde{y}_{P}\right)\right)
$$

where $g$ is a negative function defined by

$$
g\left(\tilde{y}_{P}\right)=-2+\left[\left(1-\tilde{y}_{P}\right) / S_{P}^{+}\right]=\frac{1}{\mathrm{~d} S^{+} /\left.\mathrm{d} \tilde{y}\right|_{\tilde{y}_{P}}} .
$$

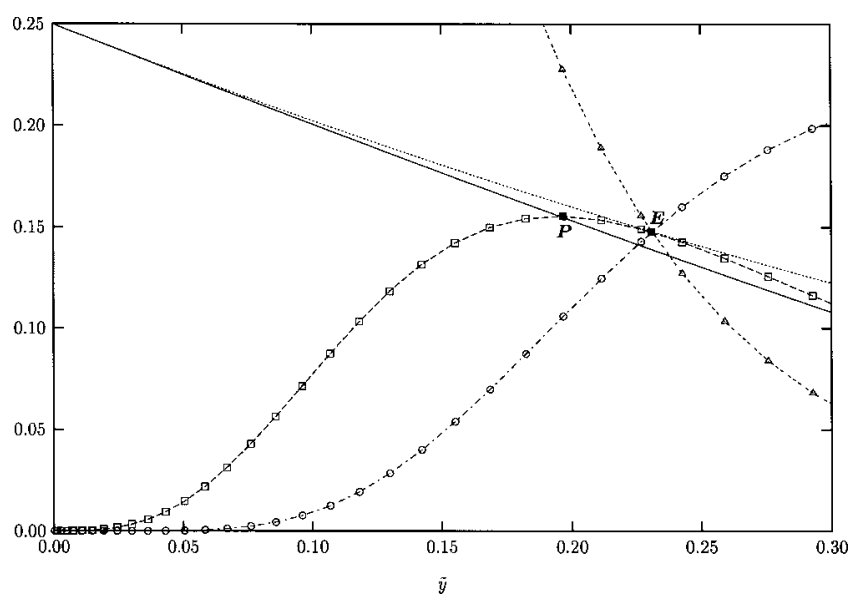

FIG. 2. Shear stresses and turbulent kinetic energy production from present DNS at $R^{+}=72 . \quad-\triangle---,\left(S^{+}\right)^{2} ;-\bigcirc \cdot-,\left(\overline{u v}^{+}\right)^{2} ; \quad-\square-, P^{+} ; \cdots, P_{E}^{+}$ [Eq. (3)]; $\longrightarrow ; P_{P}^{+}$[Eq. (9)]. 


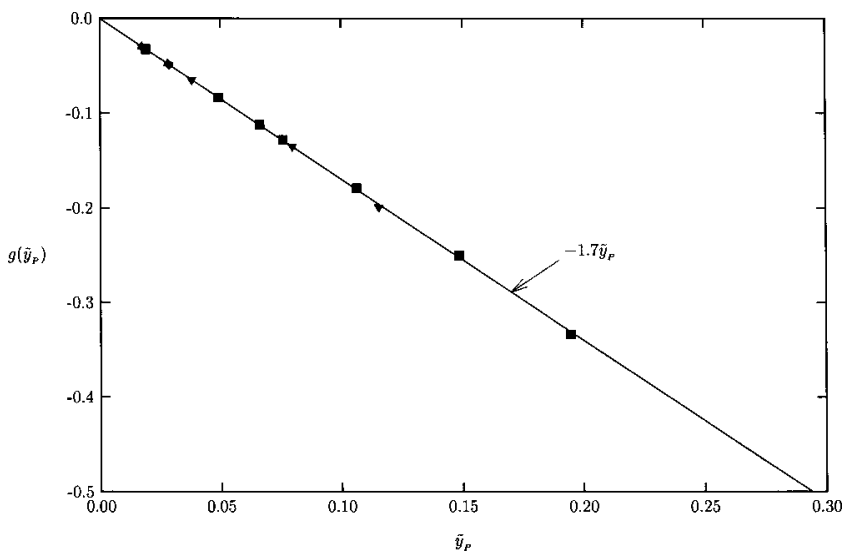

FIG. 3. Variation of $g$ with $\tilde{y}_{P}$ in channel flow;,$- g=-1.7 \tilde{y}_{P}$. The symbols are the same as in Fig. 1.

The values of this function, extracted from numerical simulations of channel flow, are plotted in Fig. 3 against $\tilde{y}_{P}$. This figure shows that $g$ decreases linearly with $\tilde{y}_{P}$ according to the fitting law $g\left(\tilde{y}_{P}\right) \approx-1.7 \tilde{y}_{P}$. Using this expression in Eqs. (8) and (7) we can deduce an evolution for the peak of production $P_{P}^{+}$as a function of its position $\tilde{y}_{P}$

$$
P_{P}^{+}=\left(\left(1-\tilde{y}_{P}\right) /\left(2-1.7 \tilde{y}_{P}\right)\right)^{2}\left(1-1.7 \tilde{y}_{P}\right) \text {. }
$$

This relation is plotted in Fig. 4 with the envelope [Eq. (3)] and the production curves for different Reynolds numbers. This figure highlights the Reynolds number dependence of the turbulence production in agreement with the equation of the envelope [Eq. (3)] and the equation of the maximum [Eq. (9)].

It is also interesting to note from Eq. (8) that the function $1 / g(\tilde{y})$ corresponds to the envelope of the mean viscous transport term $R^{+} \mathrm{d} S^{+} / \mathrm{d} y^{+}$(normalized by the pressure gradient). Since the sum of the so normalized mean turbulent and viscous transport terms is equal to -1 , the envelope of the mean turbulent transport term is $-(1+1 / g(\tilde{y}))$. The curves displayed in Fig. 5 show a good agreement between the above envelope expressions and the distributions across the channel of the transport terms for different Reynolds

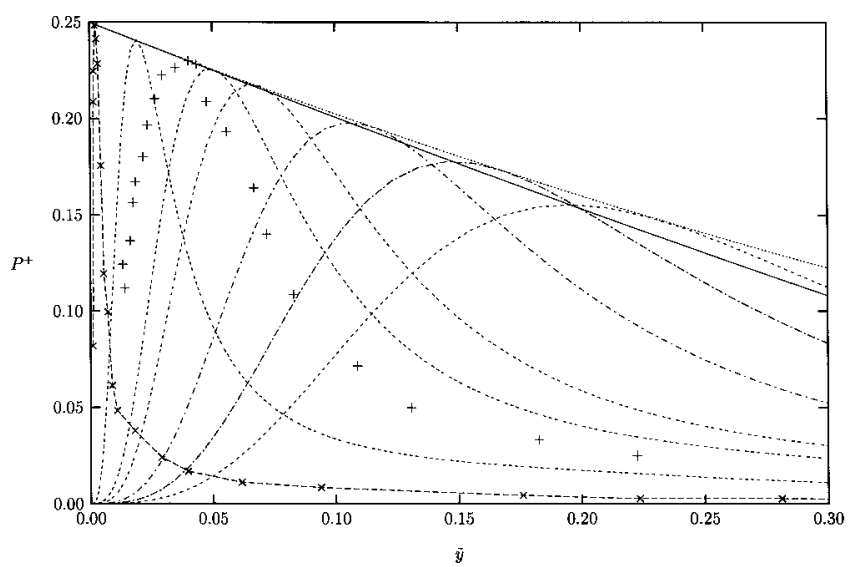

FIG. 4. Turbulent kinetic energy production in channel flow. Present study: ,$--- R^{+}=72$; - - -, $90 ;-\cdot-, 120 ;----, 180$; ----, 235; ---- -, 590. Experimental data: (+), LDV measurements of Günter et al. (Ref. 10) at $R^{+}$ =297. (-- X--) Comte-Bellot (Ref. 11), $R^{+}=4800 . \cdots, P_{E}^{+}[$Eq. (3)], $P_{P}^{+}$[Eq. (9)]. [The data from Comte-Bellot were obtained by differentiating the mean velocity profile and using Eq. (2).]

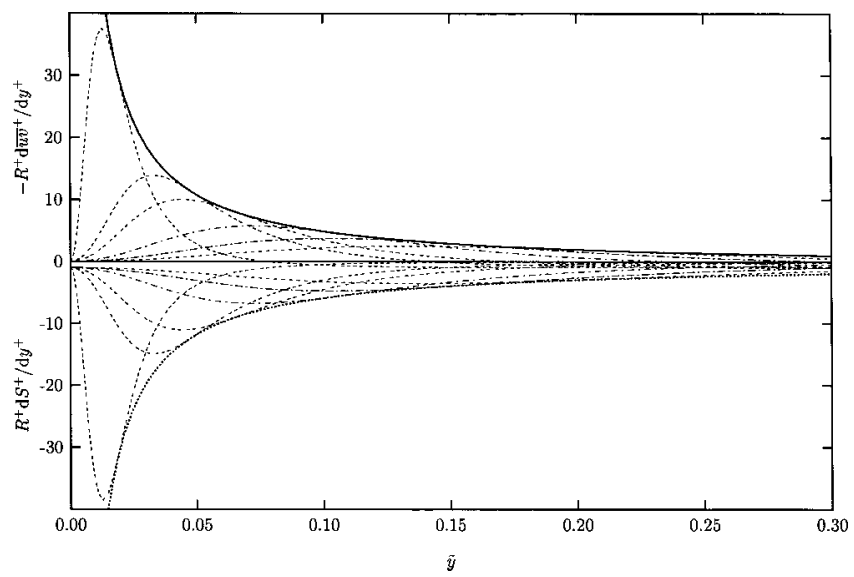

FIG. 5. Profiles of the mean transport terms in turbulent channel flow. Envelope curves: $\cdots, 1 / g(\tilde{y})$;,$--(1+1 / g(\tilde{y}))$. Other symbols are the same as in Fig. 4.

numbers. Thus, we can conclude that the peak of production coincides with the point of the envelope of the transport terms.

Sahay and Sreenivasan ${ }^{5}$ argued that the limiting behavior of $y_{P}^{+}$in conjunction with Eq. (5) implies that, for large Reynolds numbers, the position of the peak of turbulence production must coincide with the location of the extrema of the mean momentum transport terms. However, this is inconsistent with the foregoing result and with the increase without limits with Reynolds number of the normalized transport terms observed in Fig. 5. Indeed, this behavior implies that the position of the extrema of the transport terms remains always below the point of the envelope. Note that, because of the constancy of the sum of the turbulent and viscous transport terms, their extrema occur at the same position $y_{T}^{+}$.

This is obvious from Fig. 6 where are plotted, against the Reynolds number, the evolutions of $y_{P}^{+}$and $y_{T}^{+}$. This figure shows that $y_{T}^{+}<y_{P}^{+}$and brings out the asymptotic behavior for $R^{+} \rightarrow \infty$, with limiting values $y_{P_{\infty}}^{+}=11$ and $y_{T_{\infty}}^{+}=7.35$. Hence, we can clearly conclude from the above results that their statement is erroneous.

As for $y_{E}^{+}$, we derived the following empirical expressions for $y_{P}^{+}$and $y_{T}^{+}$:

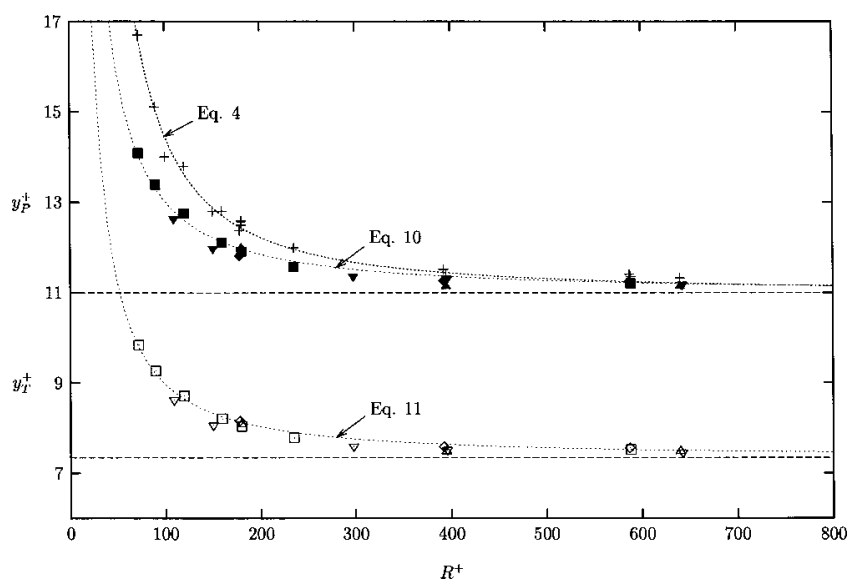

FIG. 6. Wall-normal position of the peaks of production and mean transport terms vs Reynolds number $R^{+}$. (+), $y_{E}^{+}$; filled symbols, $y_{P}^{+}$; open symbols, $y_{T}^{+}$. Symbols are the same as in Fig. 1. 


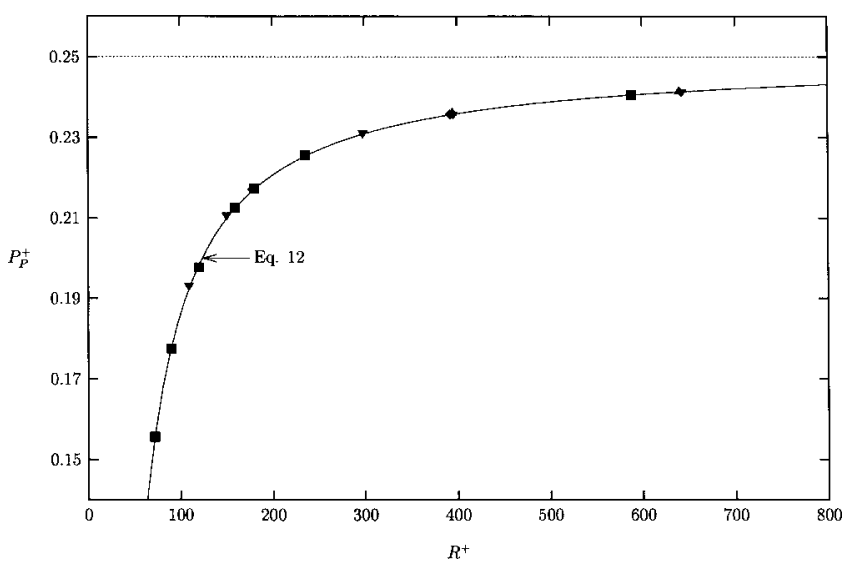

FIG. 7. Reynolds number evolution of peak production intensity in channel flow. The symbols are the same as in Fig. 1.

$$
\begin{aligned}
& y_{P}^{+}=11+638 R^{+-1.25}, \\
& y_{T}^{+}=7.35+514 R^{+-1.25} .
\end{aligned}
$$

From Eqs. (9) and (10), we obtain a relation between $P_{P}^{+}$and $R^{+}$

$$
\begin{aligned}
P_{P}^{+}= & \left(1-18.7\left(1 / R^{+}+58 / R^{+2.25}\right)\right) \\
& \times\left[\frac{1-11\left(1 / R^{+}+58 / R^{+2.25}\right)}{2-18.7 *\left(1 / R^{+}+58 / R^{+2.25}\right)}\right]^{2},
\end{aligned}
$$

allowing a good estimation of the peak of turbulence production in turbulent channel flow as shown in Fig. 7.

In conclusion, we have shown, with analytical considerations combined with DNS results, that the Reynolds number effects on the turbulence production rate in the near-wall region of channel flow are caused by the streamwise pressure gradient which enters into the mean momentum equation. Moreover, we have quantified the Reynolds number dependence of the position and intensity of the peak of turbulence production. The relations we derived can be used as a useful constraint for turbulence models.

\section{ACKNOWLEDGMENTS}

The author is grateful to Professor Marc Buffat for the use of his computer code and thanks Professor J.-N. Gence for stimulating discussions. The computing resources were provided by CINES (Centre Informatique National de l'Enseignement Supérieur), CDCSP (Centre pour le Développement du Calcul Scientifique Parallèle) at the University of Lyon 1 and CGCV (Centre Grenoblois de Calcul Vectoriel du CEA).

${ }^{1}$ R. A. Antonia, M. Teitel, J. Kim, and W. H. Browne, "Low-Reynoldsnumber effects in a fully developed turbulent channel flow," J. Fluid Mech. 236, 579 (1992).

${ }^{2}$ R. A. Antonia and J. Kim, "Low-Reynolds-number effects on near-wall turbulence," J. Fluid Mech. 276, 61 (1994).

${ }^{3}$ M. Fischer, J. Jovanović, and F. Durst, "Reynolds number effects in the near-wall region of turbulent channel flows," Phys. Fluids 13, 1755 (2001).

${ }^{4}$ F. S. Godeferd and L. Lollini, "Direct numerical simulations of turbulence with confinement and rotation," J. Fluid Mech. 393, 257 (1999).

${ }^{5}$ A. Sahay and K. R. Sreenivasan, "The wall-normal position in pipe and channel flows at which viscous and turbulent shear stresses are equal," Phys. Fluids 11, 3186 (1999).

${ }^{6}$ R. D. Moser, J. Kim, and N. N. Mansour, "Direct numerical simulation of turbulent channel flow up to $\operatorname{Re}_{\tau}=590$," Phys. Fluids 11, 943 (1999).

${ }^{7}$ H. Kawamura, K. Ohsaka, H. Abe, and K. Yamamoto, "DNS of turbulent heat transfer in channel flow with low to medium-high Prandtl number," Int. J. Heat Fluid Flow 19, 482 (1998).

${ }^{8}$ H. Kawamura, H. Abe, and Y. Matsuo, "Direct numerical simulation of turbulence by parallel computation," in Parallel Computational Dynamics (Elsevier Science, New York, 1999), pp. 3-9.

${ }^{9}$ K. Iwamoto, Y. Suzuki, and N. Kasagi, "Reynolds number effect on wall turbulence: Toward effective feedback control,' Int. J. Heat Fluid Flow 23, 678 (2002).

${ }^{10}$ A. Günter, D. V. Papavassiliou, M. D. Warholic, and T. J. Hanratty, "Turbulent flow in a channel at low Reynolds number," Exp. Fluids 25, 503 (1998).

${ }^{11}$ G. Comte-Bellot, "Contribution à l'étude de la turbulence de conduite," Ph.D. thesis, University of Grenoble, 1963 [translated by P. Bradshaw, "Turbulent flow between two parallel walls," ARC31609, FM 4102, 1969]. 